\title{
Proactive risk assessment via fuzzy approach in a decisional process of consignment stock program adoption
}

\author{
Monica Faur $^{1, *}$ and Constantin Bungău ${ }^{1}$ \\ ${ }^{1}$ University of Oradea, Engineering and Management Department, 1 Universitătii str., Oradea, \\ Romania
}

\begin{abstract}
The idea of adopting the consignment stock concept has enriched the landscape of efficient supply chains and their organizations, due to its major benefits in reducing inventory, compressing delivery time and increasing flexibility towards achieving agility and enhanced market responsiveness. The decision making process is a complex one, as besides the benefits and the economical and administrative aspects, there are also risks that must be identified, measured, assessed and managed. There is little research in the literature concerning the risks and constraints of consignment inventory implementation, while consignment contracts are widely applied in both physical and virtual supply chains. This paper introduces a model of proactive risk assessment via a fuzzy approach, allowing a sensitivity analysis of the identified risks in the matrix, in terms of probability to happen, degree of severity, impact and potential consequences, as well as mitigation. A fuzzy inference system is used to serve as assessment instrument, to fairly and more rigorously evaluate the risks, in order to avoid critical situations during or after program adoption, or even implementation failure. Fuzzy logic theory has been chosen to capture the uncertainty that usually occurs when dealing with risks and decision making. We believe that having these risk assessment insights at hand, managers and practitioners can achieve a better understanding of the challenges that come along with a new consignment program adoption, while allowing them to make the right and justified decision, in accordance with both benefit and risk considerations.
\end{abstract}

\section{Introduction}

Decisions relating to risk assessment and management frequently rely on inadequate, ambiguous, and/or subjective information [1-2]. For this reason, more research-based risk constituents along with pragmatic risk components are needed to be analysed and carefully assessed in order to prepare the implementation of a project, or to avoid critical situations [3]. For example, in industry, the risk analysis of the processes can be made by using the fuzzy rules for the code expert knowledge which belongs to the knowledge-based system [4].

* Corresponding author: monifaur@gmail.com 
An effective risk assessment, followed by an appropriate management of risks is vital to a project's success. Regardless of the project particularities or size, delivering it within budget and within the required timeframe is quite a difficult or even impossible task, if you haven't considered sufficient time and resources to identify, categorize, prioritize, assess and measure the impact of internal and external risks prior to project "go live" phase. This paper aims to present results that are part of a larger research centred on consignment stock program (CSP) adoption and implementation in a supply chain managed under lean and agile strategies [5]. The present study is focussed on risk analysis and introduces a model of proactive risk assessment via a fuzzy approach, allowing a sensitivity analysis of the identified risks in the matrix, in terms of probability of occurrence, degree of severity, impact and potential consequences.

\section{Contextual background and research motivation}

In contemporary years, the consignment stock concept has attracted increasing interests, and continuous preoccupation of both practitioners and researchers [6-9]. Dynamic environment characterized by uncertainty, expressed by market volatility and stochastic demand have pushed many organizations to adopt inventory strategies that ensure materials at hand, while reducing stock value. CSP is an efficient instrument to lower inventory cost, eliminate out of stock cases and forecast accuracy issues, as well as bringing procurement lead-times to zero, all of these being essential targets in supply chain management [10]. Moreover, CSP ensures production continuity, flexibility and increased agility, which are extremely important in dynamic and competitive markets [11]. Due to its multiple and significant advantages, the consignment inventory concept has been widely discussed in the literature in terms of fundamentals and benefits [5, 12-13], or type of contracts [14], either in case the inventory is managed by the vendor, or it is with the buyer. However, there is little research in the literature concerning the risks and constraints of consignment inventory implementation, while consignment contracts are widely applied in both physical and virtual supply chains. Furthermore, it is noted by several practitioners that the adoption and implementation of a new project in the context of a collaborative environment, CSP being one of these cases, introduces additional risks [15-16].

Risk analysis in case of CSP adoption has been prior investigated and discussed by Faur et al, (2020), [17], in terms of perceived risks associated with the challenges, creating strong barriers to the implementation process. The above mentioned study provided a quality analysis conducted by the research team through one-way ANOVA statistical method, in terms of severity and risk dispersion among different stakeholder groups. The analysis helped to also identify which of the perceived risks were just fears, feelings or human thoughts, generating problems and sometimes a pseudo perception of risk, and which of the risks had to be accounted as real. The present study continues the CSP risks analysis, identifying threats or real risks, from both internal and collaborative environment, followed by a quantitative assessment relating to risk impact, using a different and more precise approach.

\section{Data collection and research methodology}

The research data has been collected from two exploratory case studies, represented by pilot trials of CSP adoption in two different business units of a corporate organization, one of the project being successful, while the other one having a negative outcome. Identified risks, collected based on the literature review and adapted to the perceived risks delivered by the case studies, were grouped in four categories and evaluated by an expert group in a 
risk matrix template, in terms of severity, likelihood, impact and possible consequences. Then, a fuzzy inference system (FIS) was used to create a CSP risk assessment model with a higher accuracy. Mamdani FIS was chosen to assess the collected data, using Matrix Laboratory (MATLAB), version 7.0.1.24704, 2004, developed by MathWorks U.S. As a result, a risk impact score (RIS) has been obtained for each risk category and a project weighted RIS has been calculated. Based on the resulted RIS, the expert group, composed by specialists from the field, the research team and stakeholders' representatives, came with recommended actions for risk mitigation and a new assessment has been conducted in order to achieve a better project RIS.

A framework for the proposed model is presented in Figure 1.

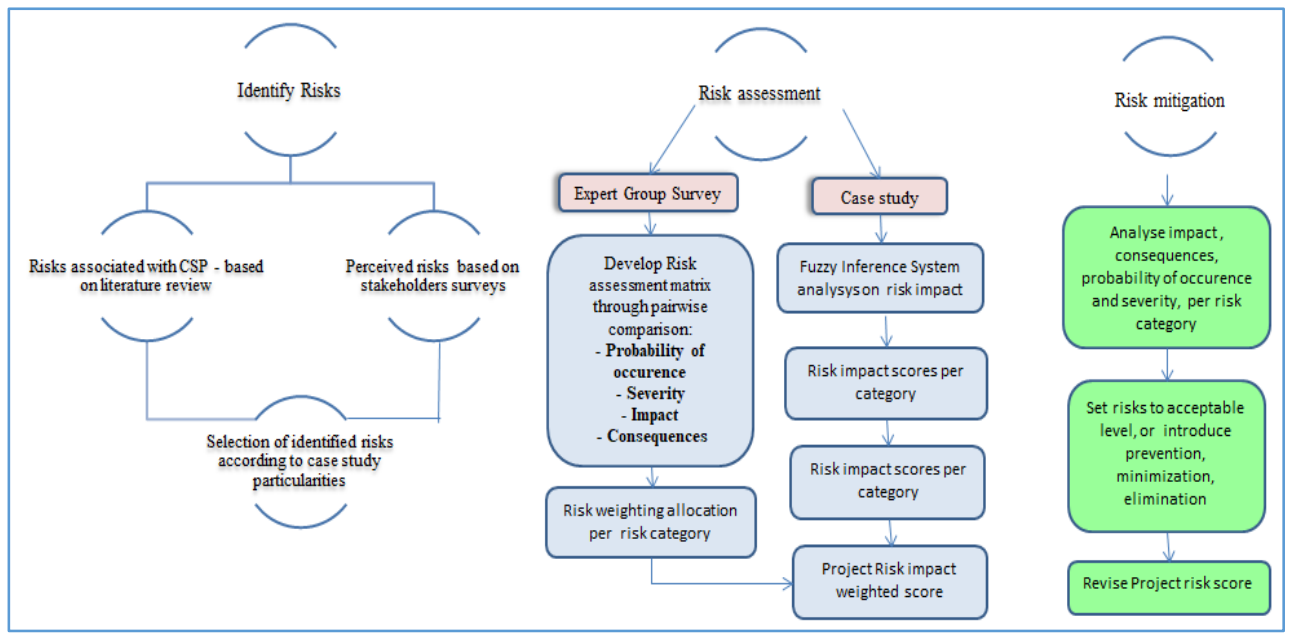

Fig. 1. The framework of the proposed model of risk assessment

\section{Risk Assessment - brief literature overview}

Risk assessment is a comprehensive operation, to appraise the possible impact of an event or of its outcome [18]. Analysing risks helps identifying effective and appropriate means and methodologies to proactively prevent, mitigate or avoid the risks [3]. A bad management of risks may result in managerial failures and in wrong decisions that neither will drive a project towards a successful implementation, nor ensure performance and company's growth.

The concept of risk assessment is addressed in the literature in different ways and in a wide range of fields, towards identifying, measuring and evaluating, in order to ensure a better control of the risks. Two well-known methodologies are mainly used for risk analysis: qualitative and quantitative. Many of the studies use qualitative methodologies, despite the fact that quantitative methodologies allow more accurate risk analyses, along with more reliable measurement for examining the risks [3]. Qualitative risk assessment tends to be more subjective, focusing on identifying risks and measuring both the impact and the likelihood of a specific risk incident that might occur during the project life cycle. Results, that are subject of interpretations, are then recorded in a risk assessment matrix, or in other risk reporting formats, in order to acknowledge the stakeholders about the possible threats and their degree of severity. On the other hand, quantitative risk assessment considers accurate numerical data, more efficient methods, robust models, or specialized software, in order to produce actionable insights. Qualitative risk analysis can be executed at any stage of the project, however, for better and more accurate results it must be 
followed by a quantitative assessment. The latter intends to clear the uncertainty surrounding the initial results from the qualitative risk analysis, quantifying as well possible outcomes and the rate of project success.

The unknown, the incertitude and vagueness concerning innovative processes, is linked not only to the implicit risk of failure, but also to the success possibility, which brings the need to adequately identify, assess and manage the risks within a new project [19], such as CSP adoption. Therefore, organizations seek for already tailored and verified strategies or models that allow to conducting early diagnosis of the involved risks [19], in order to establish certain actions, to properly manage them.

The study of the literature on the subject of risk analysing, reveals several risk evaluation approaches, widely used in the business environment, such as the balanced scorecard (BSC) [20], the failure mode effects analysis (FMEA) [21], analytic hierarchy process (AHP) [22], fault tree analysis (FTA) [23], and the risk diagnosing methodology (RDM) [24]. For instance, FMEA is a method that can be used in different fields to analyse the causes and effects of risks, take appropriate and proactive measures in order to increase systems reliability and security [21]. Another example is RDM approach, which provides strategies to support a given project towards successful implementation, by identifying and managing its potential risks [24]. The project risk is defined by several factors, not only by the risk probability to happen, risk impact and its related effects, but also by the organizations' capability to take the adequate actions, concerning each risk [24]. According to several authors [25-26], there are also other instruments that can be used to identify and analyse the risk: Decision Trees, Risk Matrix, Cause-Effect Diagrams, Structured Interviews or Surveys, Delphy's Analysis, Brainstorming Bayes Networks, among others.

Another frequently used approach in risk analysis research is multi-criteria decisionmaking method (MCDM). Fuzzy logic is also a practical tool used in situations characterised by uncertainty and where linguistic evaluations are needed. In consequence, fuzzy sets and MCDM methods are often used together [27]. Recent studies also consider the adaptive neuro-fuzzy inference system (ANFIS), which is a robust and capable model used for predictions, or risk factors assessment in contexts that generate interpretability [28]. ANFIS is functionally equivalent with Sugeno and Tsukamoto fuzzy system, but it has the capacity to adapt during a learning process [29].

The potential of fuzzy logic to assess risks impact, more precisely than human logical interpretation, is a powerful reason for taking the approach of fuzzy systems in this work. In fuzzy logic, the output appraisal is executed by a computing structure called the fuzzy inference system (FIS), which maps fuzzy inputs to the output - usually called a fuzzy set [30]. There are two commonly used fuzzy approaches: the Mamdani model and the Tagaki - Sugeno model. Mamdani approach was chosen for the present study, due to its wide acceptance, but also due to its intuitive properties and for being better adapted to inputs from human reasoning [31].

\section{Risk assessment, associated with CSP}

\subsection{Identify risks}

According to the literature review, the following risks are mentioned by different authors $[13,32-35]$ in the context of CSP adoption, from both buyer and vendor perspectives:

- Physical loss, defect or obsolete products

- Damage in the customer's warehouse

- Overstocking or duplicate inventories

- Discrepancies in inventory records, data standardisation issues 
- Selection of goods that are not suitable for CSP

- Disputes over unrecorded usages and claims

- Partner choice

- Increased inventory in the supply chain

- Delay in payments

- Accounting problems, CSP inventory tracking

- Increased labour costs due to double handling

The carried-out surveys by the project team, addressed to the stakeholders from the case studies, revealed the perceived risks that came aboard with the CSP adoption and implementation process, which are listed below:

- Inaccurate reports due to increased complexity

- Extra financial resources for training requirements

- Increased time per operation (more attention required)

- Additional human resources versus business plan (BP) - special approvals needed along with financial resources

- Extra working time - overtime constraints

- Extra storage place required by CS policy (material delimitation per each vendor)

- Lack of local legislation regarding CS concept

- Increased complexity of work (weekly reconciliations with vendors)

- Lack of coordination and correlation in case the vendor uses a different software than the buyer

- Misaligned procedures across functions

- Questioning suppliers' reliability and capabilities

All these insights have been filtered by the project team together with the expert group, resulting in the list of identified risks shown in Figure 2.

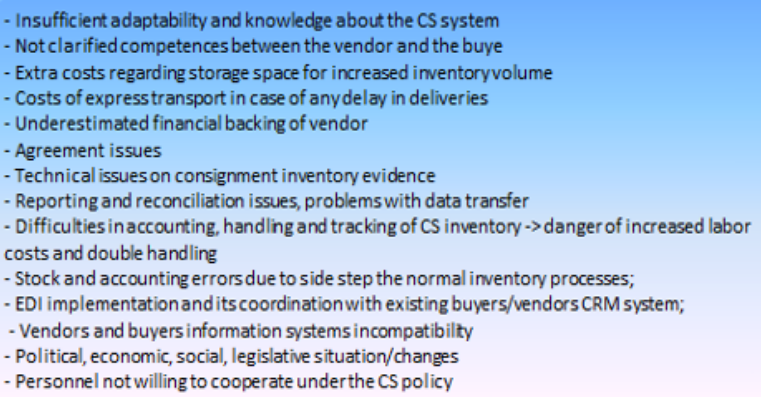

Fig. 2. Identified risks associated with CSP

\subsection{CSP risks assessment}

The identified risks have been integrated in a risk assessment matrix (Figure 3), grouped on different categories and assessed by the expert group, qualitatively, in terms of severity, likelihood, risk impact and possible consequences (Figure 4). 


\begin{tabular}{|c|c|c|c|}
\hline \multicolumn{4}{|c|}{ SCALE OF SEVERITY } \\
\hline & ACCEPTABLE & TOLERABLE & GENERALIY UNACCEPTABLE \\
\hline NOT LIKELY & Low & MEDIUM & MEDIUM \\
\hline POSSIBLE & Low & MEDIUM & HIGH \\
\hline PROBABLE & MEDIUM & HIGH & HIGH \\
\hline
\end{tabular}

Fig. 3. Risk assessment matrix

\begin{tabular}{|c|c|c|c|c|c|c|}
\hline Risk Category & \begin{tabular}{c|} 
Risk \\
no.
\end{tabular} & RISK & SEVERITY & LIKELIHOOD & \begin{tabular}{c|} 
RISK \\
IMPACT
\end{tabular} & POSSIBLE CONSEQUENCES \\
\hline \multirow{4}{*}{$\begin{array}{l}\text { 1. Internal } \\
\text { processes }\end{array}$} & R1.1. & $\begin{array}{l}\text { Missing competences on CSP } \\
\text { transactions. Training cost (TC) }\end{array}$ & Acceptable & Possible & Medium & \multirow{3}{*}{$\begin{array}{l}\text { Additional costs not included in the } \\
\text { business plan(BP). Extra financial } \\
\text { resources needed. }\end{array}$} \\
\hline & R1.2. & $\begin{array}{l}\text { Additional workload. Extra full time } \\
\text { equivalent (FTE) needed - FTE cost - (FTE) }\end{array}$ & Acceptable & Probable & Medium & \\
\hline & R1.3. & $\begin{array}{l}\text { Cost of additional information system } \\
\text { module (SAP CS module)- (CISM) }\end{array}$ & Tolerable & Probable & Low & \\
\hline & R1.4. & $\begin{array}{l}\text { Reports delays due to increased } \\
\text { complexity of work (RD) }\end{array}$ & Acceptable & Probable & Medium & $\begin{array}{l}\text { Innacurate reports; stock and } \\
\text { accounting errors }\end{array}$ \\
\hline \multirow{2}{*}{$\begin{array}{l}\text { 2. Organization } \\
\text { capabilities }\end{array}$} & R2.1. & $\begin{array}{l}\text { Storage space constraints for the } \\
\text { consigned materials (SSC) }\end{array}$ & $\begin{array}{c}\text { Generally } \\
\text { unacceptable }\end{array}$ & Probable & Medium & $\begin{array}{l}\text { Mix consigned materials with material } \\
\text { stock from other suppliers }\end{array}$ \\
\hline & $R 2.2$. & $\begin{array}{l}\text { Cost for extra storage space, to reshape } \\
\text { warehouse material flow (CESS) }\end{array}$ & Tolerable & Possible & Low & Extra financial resources requirement \\
\hline \multirow[b]{2}{*}{ 3. Regulation } & R3.1. & $\begin{array}{l}\text { Legislation compliance. Lack of local } \\
\text { legislation concerning CSP (LC) }\end{array}$ & $\begin{array}{c}\text { Generally } \\
\text { unacceptable }\end{array}$ & Possible & High & Compromise solution \\
\hline & R3.2. & $\begin{array}{l}\text { Innacurate -incomplete CSP agreement } \\
\text { (IA) }\end{array}$ & $\begin{array}{c}\text { Generally } \\
\text { unacceptable }\end{array}$ & Probable & High & $\begin{array}{l}\text { Colaboration disfunctions in several } \\
\text { areas (planning, procurement, } \\
\text { operation, accounting, sales etc) }\end{array}$ \\
\hline \multirow{4}{*}{$\begin{array}{l}\text { 4. Collaborative } \\
\text { environment }\end{array}$} & R4.1. & $\begin{array}{l}\text { Questioning suppliers reliability and } \\
\text { capability/Financial backup (SRC) }\end{array}$ & Tolerable & Possible & Medium & $\begin{array}{l}\text { Operational disfunctions, } \\
\text { discontinuities }\end{array}$ \\
\hline & R4.2. & $\begin{array}{l}\text { Questioning suppliers selection, contract } \\
\text { allocation \& segregation of duties } \\
\text { between buver and vendor (SSCA) }\end{array}$ & $\begin{array}{c}\text { Generally } \\
\text { unacceptable }\end{array}$ & Not likely & Medium & $\begin{array}{l}\text { Staff demotivation, operational issues, } \\
\text { reporting problems, stock and } \\
\text { accounting errors }\end{array}$ \\
\hline & R4.3. & $\begin{array}{l}\text { Incompatible information systems - } \\
\text { supplier-buyer (IIS) }\end{array}$ & Tolerable & Possible & Medium & $\begin{array}{l}\text { Rreporting problems, stock and } \\
\text { accounting errors }\end{array}$ \\
\hline & R4.4. & $\begin{array}{l}\text { Claims, returned goods, reconciliation } \\
\text { disputes (CRGRD) }\end{array}$ & Tolerable & Probable & Medium & $\begin{array}{l}\text { Geopardise colaboration between CS } \\
\text { partners, unilateral agreement } \\
\text { termination }\end{array}$ \\
\hline
\end{tabular}

Fig. 4. Qualitative risk assessment, by categories

The quantitative risk assessment is further performed based on a set of "If-and-Then" inference rules in line with the risk category.

The present paper includes only the quantitative assessment on risk impact, the severity and likelihood being subject of a future work. The linguistic variables, the corresponding triangular membership rules and fuzzy parameters were set for the input and output variables as shown in Figures 5,6,7,8. 


\begin{tabular}{|c|c|c|c|c|c|c|c|}
\hline Risk Category & $\begin{array}{l}\text { Risk } \\
\text { No. }\end{array}$ & Identified risks/Input variables & Notations & $\begin{array}{l}\text { Weighting } \\
(\%)\end{array}$ & Input range & $\begin{array}{l}\text { Linguistic variables, membership rules and } \\
\text { fuzzy parameters for risk impact; }\end{array}$ & $\begin{array}{c}\text { Linguistic variables } \\
\text { and output range for } \\
\text { risk impact - }[0-1]\end{array}$ \\
\hline \multirow{4}{*}{$\begin{array}{c}\begin{array}{c}\text { 1. Internal } \\
\text { processes } \\
\text { (weighting } 30 \% \text { ) }\end{array}\end{array}$} & R1.1. & $\begin{array}{c}\text { Missing competences on CSP } \\
\text { transactions. Training cost } \\
\text { (TC) }\end{array}$ & TC & $5 \%$ & {$[0-3000]$} & $\begin{array}{l}\text { TC }<2000 € \Rightarrow>\text { acceptable }\left[\begin{array}{ll}0 & 02000\end{array}\right] \\
2000 €<\mathrm{TC}<3000 € \Rightarrow \text { tolerable }\left[\begin{array}{ll}2000 & 25003000\end{array}\right] \\
\mathrm{TC}>3000 € \text { - gen. unacceptable }\left[\begin{array}{ll}2500 & 3000 \\
3000\end{array}\right.\end{array}$ & \multirow{4}{*}{$\begin{array}{l}\text { Low }[0-0.2] \\
\text { Medium }[0.3-0.6] \\
\text { High }[0.7-1]\end{array}$} \\
\hline & R1.2. & $\begin{array}{c}\text { Additional workload. Extra full } \\
\text { time equivalent (FTE) needed - } \\
\text { FTE cost - (FTE) }\end{array}$ & FTE & $7 \%$ & $\begin{array}{c}1-3 \text { FTE - } \\
{[0-3]}\end{array}$ & $\begin{array}{l}\text { FTE }=1 \Rightarrow \text { acceptable }\left[\begin{array}{lll}0 & 0 & 1.2\end{array}\right] \\
\text { FTE }=2 \Rightarrow \text { tolerable }\left[\begin{array}{llll}0.3 & 1.5 & 2.7\end{array}\right] \\
\text { FTE }=3 \Rightarrow \text { gen. unacceptable }\left[\begin{array}{lll}1.8 & 2 & 3\end{array}\right]\end{array}$ & \\
\hline & R1.3. & $\begin{array}{c}\text { Cost of additional information } \\
\text { system module ( SAP CS } \\
\text { module)-(CISM) }\end{array}$ & CISM & $15 \%$ & {$[0-5000]$} & $\begin{array}{l}\text { CISM }<4000 € \Rightarrow>\text { acceptable }\left[\begin{array}{lll}0 & 0 & 4000\end{array}\right] \\
4000 €<C I S M<5000 € \Rightarrow \text { tolerable }\left[\begin{array}{llll}2500 & 4000 \\
5000\end{array}\right] \\
\text { CISM } 55000 € \text { - unacceptable }\left[\begin{array}{lll}4500 & 5000 & 5000\end{array}\right]\end{array}$ & \\
\hline & R1.4. & $\begin{array}{l}\text { Reports delays due to } \\
\text { increased complexity of work } \\
\text { (RD) }\end{array}$ & $\mathrm{RD}$ & $3 \%$ & $\begin{array}{l}\text { Max } 3 \text { days } \\
\text { delay }-[0-3]\end{array}$ & $\begin{array}{l}\mathrm{RDD}=\text { acceptable }\left[\begin{array}{lll}0 & 0 & 1.2\end{array}\right] \\
\mathrm{RDD}=\text { tolerable }\left[\begin{array}{lll}0.9 & 1.5 & 2\end{array}\right] \\
\mathrm{RDD}=\text { unacceptable }\left[\begin{array}{lll}2.5 & 2.5 & 3\end{array}\right]\end{array}$ & \\
\hline
\end{tabular}

Fig. 5. Linguistic variables, membership rules and fuzzy parameters for "Internal processes" category

\begin{tabular}{|c|c|c|c|c|c|c|c|}
\hline Risk Category & $\begin{array}{l}\text { Risk } \\
\text { No. }\end{array}$ & Identified risks/Input variables & Notations & $\begin{array}{c}\text { Weighting } \\
(\%)\end{array}$ & Input range & $\begin{array}{l}\text { Linguistic variables and membership rules for } \\
\text { risk impact; output range - [0-1] }\end{array}$ & 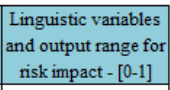 \\
\hline \multirow{2}{*}{$\begin{array}{l}\text { 2. Organizational } \\
\text { capabilities } \\
\text { (weighting 20\%) }\end{array}$} & R2.1. & $\begin{array}{l}\text { Storage space constraints for } \\
\text { the consigned materials (SSC) }\end{array}$ & SSC & $10 \%$ & $\begin{array}{c}\text { Actual = } \\
400 \text { sqm - CS } \\
\text { optim- } \\
700 \text { sqm [0-10] }\end{array}$ & $\begin{array}{l}\text { If } S=400 \mathrm{sqm} \Rightarrow>\text { unacceptable }\left[\begin{array}{lll}0.7 & 0.81\end{array}\right] \\
\text { If } \mathrm{S}=500-600 \text { sqm } \Rightarrow>\text { reasonable }\left[\begin{array}{lll}0.1 & 0.4 & 0.7\end{array}\right] \\
\text { If } \mathrm{S}=700 \mathrm{sqm} \Rightarrow>\text { optimal }\left[\begin{array}{lll}0 & 0.2 .2\end{array}\right]\end{array}$ & \multirow{2}{*}{$\begin{array}{c}\text { Low }[0-0.2] \\
\text { Medium }[0.3-0.6] \\
\text { High }[0.7-1]\end{array}$} \\
\hline & R2.2. & $\begin{array}{c}\text { Cost for extra storage space, to } \\
\text { reshape warehouse material } \\
\text { flow (CESS) }\end{array}$ & CESS & $10 \%$ & [0-500] & $\begin{array}{l}\text { CESS }<300 € \Rightarrow \text { acceptable }\left[\begin{array}{lll}0 & 0 & 0.2\end{array}\right] \\
300 €<\text { CESS }<500 € \Rightarrow \text { reasonable }\left[\begin{array}{lll}0.1 & 0.4 & 0.8\end{array}\right] \\
\text { CESS }>500 € \Rightarrow \text { high }\left[\begin{array}{lll}0.8 & 0.9 & 1\end{array}\right]\end{array}$ & \\
\hline
\end{tabular}

Fig. 6. Linguistic variables, membership rules and fuzzy parameters for "Org. capablities" category

\begin{tabular}{|c|c|c|c|c|c|c|c|}
\hline Risk Category & $\begin{array}{l}\text { Risk } \\
\text { No. }\end{array}$ & Identified risks/Input variables & Notations & $\begin{array}{l}\text { Weighting } \\
(\%)\end{array}$ & Input range & $\begin{array}{l}\text { Linguistic variables, membership rules and fuzzy } \\
\text { parameters for risk impact; }\end{array}$ & $\begin{array}{c}\text { Linguistic variables } \\
\text { and output range for } \\
\text { risk impact - }[0-1]\end{array}$ \\
\hline Risk Category & $\begin{array}{l}\text { Risk } \\
\text { No. }\end{array}$ & Identified risks/Input variables & Notations & $\begin{array}{l}\text { Weighting } \\
(\%)\end{array}$ & Input range & $\begin{array}{l}\text { Linguistic variables and membership rules for } \\
\text { risk impact; output range - }[0-1]\end{array}$ & $\begin{array}{c}\text { Linguistic variables } \\
\text { and output range for } \\
\text { risk impact - }[0-1]\end{array}$ \\
\hline \multirow{2}{*}{$\begin{array}{c}\text { 3. Regulation } \\
\text { (weighting 15\%) }\end{array}$} & R3.1. & $\begin{array}{c}\text { Legislation compliance. Lack of } \\
\text { local legislation concerning } \\
\text { CSP (LC) }\end{array}$ & LC & $10 \%$ & {$[0-10]$} & 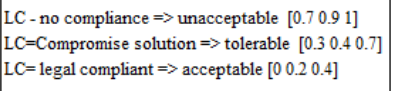 & \multirow{2}{*}{$\begin{array}{l}\text { Low }[0-0.2] \\
\text { Medium }[0.3-0.6] \\
\text { High }[0.7-1]\end{array}$} \\
\hline & R3.2. & $\begin{array}{c}\text { Innacurate - incomplete CSP } \\
\text { agreement }(\mathrm{IA})\end{array}$ & IA & $5 \%$ & {$[0-100 \%]$} & $\begin{array}{l}\text { incomplete } / \text { inaccurate }=>\text { unacceptable }\left[\begin{array}{lll}0.8 & 0.9 & 1\end{array}\right] \\
\text { less complete } \Rightarrow>\text { tolerable }\left[\begin{array}{lll}0.3 & 0.5 & 0.7\end{array}\right] \\
\text { accurate \& complete }=>\text { acceptable }\left[\begin{array}{lll}0 & 0.2 & 0.2\end{array}\right]\end{array}$ & \\
\hline
\end{tabular}

Fig. 7. Linguistic variables, membership rules and fuzzy parameters for "Regulation" category

\begin{tabular}{|c|c|c|c|c|c|c|c|}
\hline Risk Category & $\begin{array}{l}\text { Risk } \\
\text { No. }\end{array}$ & Identified risks/Input variables & Notations & $\begin{array}{l}\text { Weighting } \\
(\%)\end{array}$ & Input range & $\begin{array}{l}\text { Linguistic variables and membership rules for } \\
\text { risk impact; output range - }[0-1]\end{array}$ & $\begin{array}{c}\text { Linguistic variables } \\
\text { and output range for } \\
\text { risk impact - }[0-1] \\
\end{array}$ \\
\hline \multirow{4}{*}{$\begin{array}{l}\text { 4. Collaborative } \\
\text { environment } \\
\text { (weighting } 35 \% \text { ) }\end{array}$} & R4.1. & $\begin{array}{l}\text { Questioning suppliers } \\
\text { reliability and capability / } \\
\text { Financial backup (SRC) }\end{array}$ & SRC & $9 \%$ & $\begin{array}{l}\text { at least } 3 \\
\text { reliable supp. } \\
{[0-10]}\end{array}$ & $\begin{array}{l}3 \text { reliable supp. } \Rightarrow>\text { acceptable }\left[\begin{array}{lll}0 & 0.2 & 0.2\end{array}\right] \\
2 \text { reliable supp. } \Rightarrow \text { tolerable }\left[\begin{array}{lll}0.3 & 0.35 & 0.6\end{array}\right] \\
1 \text { supp., no backup } \Rightarrow>\text { unacceptable }\left[\begin{array}{lll}0.7 & 0.8 & 1\end{array}\right]\end{array}$ & \multirow{4}{*}{$\begin{array}{l}\text { Low }[0-0.2] \\
\text { Medium }[0.3-0.6] \\
\text { High }[0.7-1]\end{array}$} \\
\hline & R4.2. & $\begin{array}{c}\text { Questioning suppliers } \\
\text { selection, contract allocation \& } \\
\text { segregation of duties between } \\
\text { buyer and vendor (SSCA) }\end{array}$ & SSCA & $9 \%$ & {$[0-10]$} & $\begin{array}{l}\text { correct/ transparent allocation =>acceptable }[0.2 \\
0.2] \\
\text { questionable allocation=>less tolerable }\left[\begin{array}{ll}0.4 & 0.6 \\
0.8\end{array}\right] \\
\text { incorrect selection \& duties } \\
\text { allocation=>unacceptable }\left[\begin{array}{ll}0.7 & 0.81\end{array}\right]\end{array}$ & \\
\hline & R4.3. & \begin{tabular}{|c|} 
Incompatible information \\
systems - supplier-buyer (IIS)
\end{tabular} & IIS & $10 \%$ & {$[0-10]$} & $\begin{array}{l}\text { incompatible }\left[\begin{array}{lll}0.7 & 0.8 & 1\end{array}\right] \\
\text { less incompatible }\left[\begin{array}{lll}0.3 & 0.5 & 0.6\end{array}\right] \\
\text { compatible }\left[\begin{array}{lll}0 & 0.2 & 0.2\end{array}\right]\end{array}$ & \\
\hline & R4.4. & $\begin{array}{l}\text { Claims, returned goods, } \\
\text { reconciliation disputes } \\
\text { (CRGRD) }\end{array}$ & CRGRD & $7 \%$ & {$[0-10]$} & 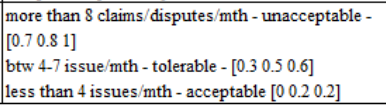 & \\
\hline
\end{tabular}

Fig. 8. Linguistic variables, membership rules and fuzzy parameters for "Collaborative environment" category

The risk assessment input variables have three levels: acceptable, tolerable and generally unacceptable, while the output variable (risk impact) was defined by certain range intervals, corresponding to the attributes: low, medium and high. Weighting allocated by 
the expert group per both individual risk and risks category, are also shown in the above figures.

\section{Results and discussions}

The FIS enables a composition of the inputs using fuzzy set theory, fuzzy 'if-then' triangular functions and fuzzy interpretation according to the set rules, in order to get to the output; more explicitly, it involves fuzzification of the input variables (transforms the crisp input data into fuzzy sets), rules evaluation, aggregation of the rule outputs and at the end, the defuzzification, meaning the extraction of a crisp value which best represents a fuzzy set[36]. Amid several defuzzification methods found in the literature, the centroid approach was chosen, given its wide use for this kind of applications. Several simulations have been performed to assess the behaviour of risks within each category. Figure 9 illustrates FIS surfaces of risk impact corresponding to "Internal processes" risk category.
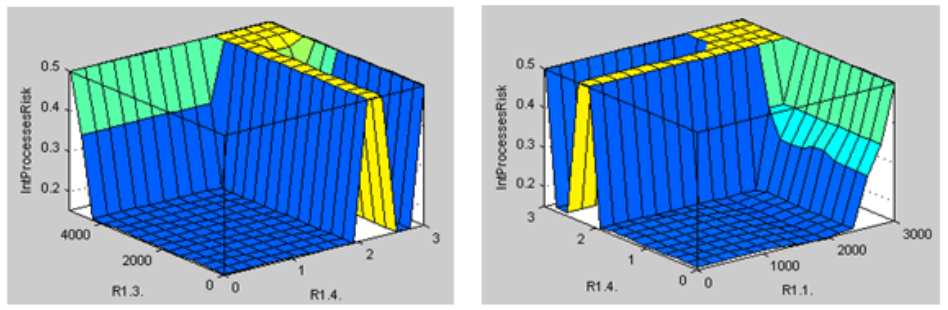

Fig. 9. FIS surfaces for different simulations of risk impact within "Internal processes" category

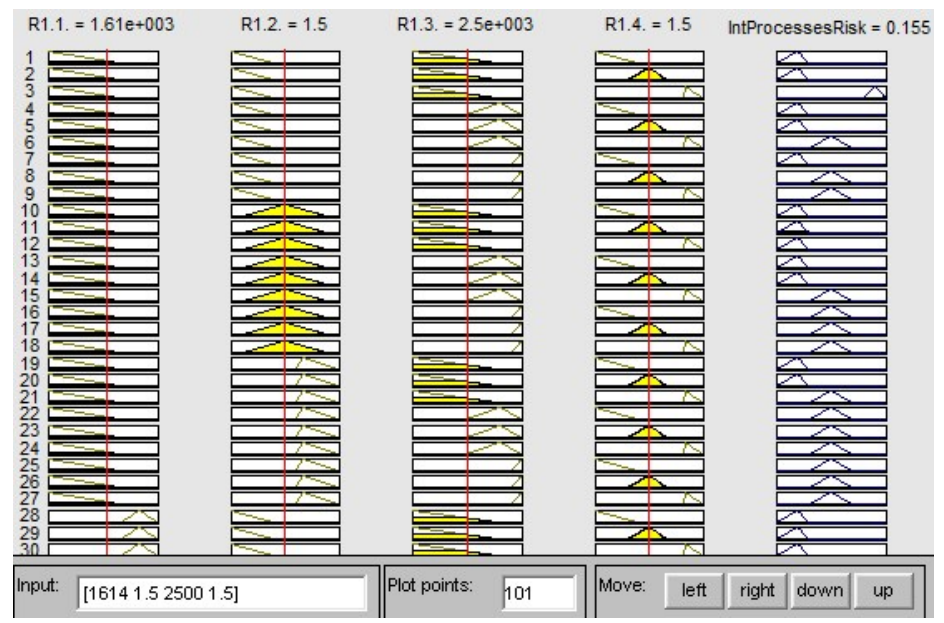

Fig. 10. FIS rule viewer for 'Internal processes' category; a suitable output value $=0.155$

The degree of the risk impact, obtained from each FIS correspondent to a category, has been defined as risk impact score (RIS). The total project RIS has been calculated as a weighted average of the RIS per categories, as per below:

$$
R I S_{\text {project }}=R I S_{\text {Int.procs }} \cdot \omega_{\text {Int.proc }}+R I S_{\text {Org.cap. }} \cdot \omega_{\text {Org.cap. }}+R I S_{\text {Reg. }} \cdot \omega_{\text {Reg }}+R I S_{\text {Colab. }} \cdot \omega_{\text {Colab }}
$$

where 


$$
\omega_{\text {Int.proc }}+\omega_{\text {Org.cap. }}+\omega_{\text {Reg }}+\omega_{\text {Colab }}=1
$$

So, in our case,

$$
R I S_{\text {project }}=0.155 \cdot 0.3+0.685 \cdot 0.2+0.573 \cdot 0.15+0.462 \cdot 0.35=0.431
$$

The obtained score is qualified as medium risk with regards to CSP implementation. As a result, several corrective actions recommended by the expert group have been taken (Figure 11), and upon FIS reiterations per categories, risks have been reduced, obtaining

\begin{tabular}{|c|c|c|c|c|c|}
\hline Risk Category & $\begin{array}{c}\text { Risk } \\
\text { no. }\end{array}$ & RISK & $\begin{array}{c}\text { RISK } \\
\text { MMPACT }\end{array}$ & RECOMAIENDED ACTION(S) & $\begin{array}{c}\text { RISK IMPACT upon } \\
\text { taken actions } \\
\end{array}$ \\
\hline \multirow{4}{*}{$\begin{array}{l}\text { 1. Internal } \\
\text { processes }\end{array}$} & R1.1. & $\begin{array}{l}\text { Missing competences on CSP } \\
\text { transactions. Training cost (TC) }\end{array}$ & Medium & \multirow{3}{*}{$\begin{array}{l}\text { BP adjustment requirements, in terms of FTE } \\
\text { and financial resources allocation }\end{array}$} & Low \\
\hline & R1.2. & $\begin{array}{l}\text { Additional workload. Extra full time } \\
\text { equivalent (FTE) needed - FTE cost - (FTE) }\end{array}$ & Medium & & Low \\
\hline & R1.3. & $\begin{array}{l}\text { Cost of additional information system } \\
\text { module (SAP CS module) - (CISM) }\end{array}$ & Low & & Low \\
\hline & R1.4. & $\begin{array}{l}\text { Reports delays due to increased } \\
\text { complexity of work (RD) }\end{array}$ & Medium & $\begin{array}{l}\text { Additional FTE allocation; ensure assistance } \\
\text { and counceling }\end{array}$ & Low \\
\hline \multirow{2}{*}{$\begin{array}{l}\text { 2. Organization } \\
\text { capabilities }\end{array}$} & R2.1. & \multirow{2}{*}{$\begin{array}{l}\text { Storage space constraints for the } \\
\text { consigned materials (SSC) } \\
\text { Cost for extra storage space, to } \\
\text { reshape warehouse material flow } \\
\text { inrmel }\end{array}$} & Medium & $\begin{array}{l}\text { Re-thing stock dimensions for all materials } \\
\text { in place, segregate materials, instruct staff }\end{array}$ & Low \\
\hline & $R 2.2$. & & Low & $\begin{array}{l}\text { Reshape warehouse material flow }+B P \\
\text { adjustments }\end{array}$ & Low \\
\hline \multirow{2}{*}{ 3. Regulation } & R3.1. & $\begin{array}{l}\text { Legislation compliance. Lack of local } \\
\text { legislation concerning CSP (LC) }\end{array}$ & High & $\begin{array}{l}\text { Legal and Financial functions research in the } \\
\text { field; finding a compromise solution; } \\
\text { carrefully drafting CS partners agreements }\end{array}$ & Medium \\
\hline & R3.2. & $\begin{array}{l}\text { Innacurate-incomplete CSP } \\
\text { agreement (IA) }\end{array}$ & High & $\begin{array}{l}\text { Draft the agreement under Legal dpt. } \\
\text { surveillance and with stakeholders } \\
\text { involvement }\end{array}$ & Low \\
\hline \multirow{4}{*}{$\begin{array}{l}\text { 4. Collaborative } \\
\text { environment }\end{array}$} & R4.1. & $\begin{array}{l}\text { Questioning suppliers reliability and } \\
\text { capability/ Financial backup (SRC) }\end{array}$ & Medium & $\begin{array}{l}\text { Ensure supplier back-up and correct } \\
\text { supplliers evaluation across the selection } \\
\text { nrnrace }\end{array}$ & Medium \\
\hline & R4.2. & $\begin{array}{l}\text { Questioning suppliers selection, } \\
\text { contract allocation \& segregation of } \\
\text { duties between buver and vendor }\end{array}$ & Medium & $\begin{array}{l}\text { Ensure transparency within suppliers } \\
\text { selection process and clarify duties } \\
\text { segregation }\end{array}$ & Low \\
\hline & R4.3. & $\begin{array}{l}\text { Incompatible information systems - } \\
\text { supplier-buyer (IIS) }\end{array}$ & Medium & $\begin{array}{l}\text { Technical know-how and expertize from } \\
\text { consultants; ensure systems compatibility }\end{array}$ & Low \\
\hline & R4.4. & $\begin{array}{l}\text { Claims, returned goods, reconciliation } \\
\text { disputes (CRGRD) }\end{array}$ & Medium & $\begin{array}{l}\text { Ensure mutual agreement clauses and agree } \\
\text { certain procedures \& responsibilities }\end{array}$ & Medium \\
\hline
\end{tabular}
$R I S_{\text {project }}=0.196$, which brings the project risks in the "low risk" range.

Fig. 11. Recommended actions and risk impact upon correction implementation

\section{Conclusions}

The goal of the risk assessment is to provide effective strategies to proactively confront the risks and manage possible negative outcomes, eventually to better allocate and commit business resources. Risk evaluation is always characterized by uncertainty, ambiguity and inevitably dependent on human perception. In this regard, a risk analysis has been conducted on the subject of CSP adoption and a risk assessment model has been proposed based on the insights from the literature review and from two case studies, with the help of an expert group. The proposed model, based on Mamdani FIS, demonstrates to ensure a risk level measurement with a higher accuracy. Based on this approach, the stakeholders would be able to appropriately anticipate and respond to risks.

A project risk impact score has been generated by the system, once the risks have been identified and grouped in categories by the expert group. An additional RIS iteration has been run, after risk mitigation actions have been implemented. The result shows that the risks can be reduced if properly addressed. Furthermore, the subjectivity problem concerning risk assessment and also possible biases associated with human perception are eliminated by integrating fuzzy logic approach in the assessment model.

CSP implies always a collaborative context, therefore, the model is suitable to comparing different impact scores and to evaluate which of the risks has higher impact on the project, the internal ones, or the ones derived from the partners, giving right directions 
for corrective actions and documented decisions. The proposed model provides a higher level of accuracy, precision and reliability in terms CSP risk assessment.

Usually, in a supply chain governed by lean and agile strategies, operational management preoccupation is to continuously improve the system in terms of efficiency and increase the organization's capability to quickly react to unpredictable market demands, sometimes neglecting the associated risks. CSP complies with operational management goals as long as risks are considered and correctly assessed. Consequently, the management teams should either take advantage of the risk assessment models, as the one described in this paper, or cooperate with researchers from academic environment in order to adapt and improve the models according to their requirements and factors that influence decisions.

The model can be also used in parallel with a regular risk assessment performed by the practitioners (such as BSC), in scope of comparing the results.

We believe that having these risk assessment insights at hand, managers and practitioners can achieve a deeper understanding of the challenges that come along with a new consignment program adoption, while allowing them to make the right and justified decision, in accordance with both benefit and risk considerations.

\section{References}

1. G. Wood, R.C.T. Ellis, Eng. Constr. Archit. Manag. 10, 254-262 (2003)

2. I. Dikmen, M.T. Birgonul, A.E. Arikan, Proc. of the 20th Annual Conf. of Assoc. of Researchers in Constr. Management, Edinburgh, 1145-1154 (2004)

3. J.M. Kim, T. Kim, S. Ahn, Sustainability 12, 5316 (2020)

4. A. Sipos, Sustainability 12(23), 10205-10221, (2020)

5. M. Faur, C. Bungău, Supply Chain 'Leagility' through Adopting Consignment Stock Strategy in Manufacturing Companies, RMEE - 6th Int. Management Conference (2018)

6. T. Avinadav, T. Chernonog, and Y. Perlman, Int. J. Prod. Econ. 168, 31-40 (2015).

7. Y. Fang, Y.-Y. Wang, Z. Hua, J. Oper. Res. Soc. 67(5), 770-785 (2016)

8. J. Ru and Y. Wang, Eur. J. Oper. Res., 201(3), 760-769 (2010)

9. Z. Wu, D. Chen, H. Yu, Int. J. Gen. Syst., 45(5), 502-516 (2016)

10. M. Faur C. Bungău, Annual Sess. Scientific Papers- IMT Oradea, 291-295 (2019)

11. M. Faur, C. Bungău, F. Blaga, I.C. Gherghea, A. Pop, The organisation readiness index, as a decision support tool for consignment stock program adoption in a 'leagile' supply chain, 7th RMEE Management Conference, Cluj- Napoca (2020)

12. G. Valentini and L. Zavanella, Int. J. Prod. Ec., 81-82: 215-224 (2003)

13. D. Battini, Int. J. Prod. Res. 48(7), 2055-2079 (2010)

14. W. Hu, Y.J. Li, W. Wang, Benefit and risk analysis of consignment contracts, Ann. Op. Res. 257, 641-659, (2017)

15. L. M. Camarinha-Matos and A. Abreu, A contribution to understand collaboration benefits, Emerging Sol. Future Manufact. Syst., Springer, (2004)

16. M. Faur and C. Bungău., Outsourcing towards greater agility by investigating decoupling points in leagile supply chains, Int. Manuf. Sci. and Ed. Conf., Sibiu, MATEC Web of Conferences 290, 07006 (2019)

17. M. Faur, A. Sipos, C. Bungău, I. C. Gherghea, Ann. Sess. of Sci. Papers, IMT Oradea 19, 153 $160(2020)$

18. C. Bao, D. Wu, J. Li, IEEE Transactions on Fuzzy Systems 27(5), 1126-1138 (2018)

19. H.L. Madsen, J. Clean. Prod. 247, 119-102 (2020)

20. J. Keizera, J. Halman, M. Song, M., J. Prod. Innov. Manag. 19, 213-232 (2002)

21. C.H. Wang, IEEE Int. Conf. Ind. Eng. and Eng. Mngm., Singapore, 1275-1278 (2011)

22. H. Liu, X. Chen, C. Duan, Y. Wang, Comput Ind Eng 135, 881-897 (2019)

23. T.L. Nguyen, M.H. Shu, B.M. Hsu, Sustainability 8(9), 939 (2016) 
24. A. Erdil, E. Tacgin, Fibres Textiles Eastern Euro 26(6), 30-42 (2018)

25. G. Monahan, Enterprise Risk Management: A Methodology for Achieving Strategic Objectives (John Wiley \& Sons, 2008)

26. R. J. Chapman, Simple Tools and Techniques for Enterprise Risk Management, 2nd Ed. (John Wiley \& Sons, 2011)

27. R. Shariat, Roozbahani A, Ebrahimian, Sci Total Environ 647, 1468-1477 (2019)

28. Z. Ebrahimi-Khusfi, R. Taghizadeh-Mehrjardi and A.R. Nafarzadegan, Env. Sci and Poll. Res., 28(6), 6796-6810 (2021)

29. A. Pop, Nonconventional Tech. Rev., 53-57 (2017)

30. J. Jang, C. Sun, E. Mizutani, Neuro-fuzzy and soft computing: A computational approach to learning and machine intelligence, 1st Ed. (Prentice Hall, New Jersey, 1997)

31. C. Harris, C. Moore, and M. Brown, Intelligent control: Aspects of Fuzzy Logic and Neural Nets. 1st Ed. (World Sci. Pub., Singapore, 1993)

32. G. Richards, S. Grinsted, The Logistics and Supply Chain Toolkit: Over 100 Tools and Guides for Supply Chain, Transp., Wh and Inv. Mngm., 2nd Ed. (Kogan Page, 2016)

33. B., R. Sarker, Int. J. Prod. Ec. 155, 52-67 (2014)

34. S. K. Zahran, M. Y. Jaber, S. Zanoni, L. E. Zavanella, Comp.\& Ind. Eng. 87, 491-505 (2015)

35. T. Wild, Best Practice in Inventory Management, 3rd Ed. (Routledge, 2017)

36. T. Tettey, T. Marwala, Modelling Conflict: Knowledge Extraction using Bayesian Neural Network and Neuro-fuzzy Models, https://www.researchgate.net/publication/267381022, (ResearchGate, 2016) 